\title{
The First - Passage Time Moment of the GNP Diffusion Process to a Determined Value
}

\author{
Mohammad Zainal and Anwar Alshriaan \\ Kuwait University, Collage of Business Administration \\ P. O. Box 5486, Safat 13055, Kuwait.
}

\begin{abstract}
This paper describes an accurate method of approximating the moments of the first-passage time for the birth and death Gross National Product GNP diffusion process when the GNP is a determined value or constant absorbing barrier. This was done by approximating the differential equations by equivalent difference equations.

Keywords: First-passage time, Birth and death GNP diffusion process, Constant absorbing barrier, Difference equations.
\end{abstract}

\section{Introduction}

First-passage time plays an important role in the area of applied probability theory especially in stochastic modeling. Several examples of such problems are the extinction time of absorbing process, the exogenous factors in economics, or the cycle lengths of a certain vehicle actuated traffic signals. More specifically, the first-passage time to a moving barrier for diffusion and other Markov processes arises in economics modeling (cf. Gutierrez et.al.(1997), (1999)), in biological modeling (cf. Ewens (1979)), in statistics (cf. Darling and Siegert (1953) and Durbin (1971)) and in engineering (cf. Blake and Lindsey (1973)).

Many important results related to the first-passage time have been studied from different points of view by different authors. For example, McNeil (1970) has derived the distribution of the integral functional $W_{x}=$ $\int_{g}^{T_{x}}\{X(t)\} d t$ where $T_{x}$ is the first-passage time to the origin in a general birth and death process with $X(o)=x$ and $g(i)$ is an arbitrary function. And, Iglehart (1965), McNeil and Schach (1973) have shown a number of classical birth and death processes upon taking diffusion limits to asymptotically approach the Ornstein-Uhlenbeck (O. U.).

Many properties such as a first-passage time to a barrier, absorbing or reflecting, located some distance from an initial starting point of the $\mathrm{O}$. U. process and the related diffusion process such as the case of the first passage time of a Wiener process to a linear barrier is a closed form expression for the density available is discussed in Cox and Miller (1965). Gutierrez et. al. (1999) presented a methodology to build a log normal

Corresponding author: Mohammad Zainal, Kuwait University, Collage of Business Administration, P. O. Box 5486, Safat 13055, Kuwait. E-mail: zainal@cba.edu.kw.

Anwar Alshriaan, Kuwait University, Collage of Business Administration, P. O. Box 5486, Safat 13055, Kuwait. E-mail: alshriaan@cba.edu.kw. 
diffusion process with exogenous factors that models economic variables. They studied the problem of forecasting the first passage time and applied to the GNP of Spain. Thomas (1975) describes some mean first-passage time approximation for the Orenstein-Uhlenbeck process. Tuckwell and Wan (1984) have studied the first-passage time of a Markov process to moving barriers as a first-exit time for a vector whose components include the process and the barrier. Also, others such as, Karlin and Taylor (1981), Ferebee (1982), Alawneh and Aleideh (2002), etc have discussed the first passage time from different points of view. In particular, Alawneh and Aleideh (2002) have discussed the problem of finding the moments of the first-passage time distribution for the Orenstein-Uhlenbeck process with a single absorbing barrier using the method of approximating the differential equations by difference equations.

In this paper, we consider the birth and death GNP diffusion process and study the first-passage time for such a process to a fixed determined value single constant absorbing barrier. More specifically, the moment approximations are derived using the method of difference equations.

\section{First-Passage Moment Approximation to the GNP Diffusion Process}

Let $\{X(t): t \geq o\}$ be the birth and death diffusion process with infinitesimal mean $b x$ and variance $z \alpha x$ starting at some $x_{o}>o$, where $b$ and $a$ are the drift and the diffusion coefficients respectively and satisfies the Ito stochastic differential equation

$$
d X(t)=b X(t) d t+\sqrt{2 a X(t)} d W(t)
$$

where $\{W(t) ; t \geq 0\}$ is a standard Wiener process with zero mean and variance $\sigma^{2} t$. Assume that the existence and uniqueness conditions are satisfied (cf. Gihman and Skorohod (1972)).

Let $\{Y(t): t \geq 0\}$ be the single constant absorbing barrier (fixed determined value of the GNP) $Y(t)=\mathrm{k}$, with $Y(o)=k$, or equivalently;

$$
\frac{d Y(t)}{d t}=0
$$

Thus the first-passage time of the GNP diffusion process $X(t)$ to a fixed determined value or a constant single absorbing barrier $Y(t)=k$ by the random variables

$$
T_{x}=\inf \{t \geq 0: x(t) \geq k\}
$$

with probability density function $g\left(t ; x_{o}\right)=-\frac{d}{d t} \int_{-\infty}^{k} P\left(x_{o}, x ; t\right) d t$. Here $p\left(x_{o}, x ; t\right)$ is the probability density function of $X(t)$. Letting $M_{n}\left(x_{o}, k ; t\right) ; n=1,2,3, \ldots .$. , be the $n-t h$ moment of the first-passage time $T_{x}$ to a constant barrier $k$, i.e.

$$
M_{n}\left(x_{o}, k ; t\right)=E\left(T_{x}^{n}\right) ; n=1,2,3, \ldots
$$

It follows from the forward Kolmogorov equation that the $n^{\text {th }}$ moment of $T_{y}$ must satisfy the ordinary differential equation

$$
\operatorname{ax} M_{n}^{\prime \prime}\left(x_{o}, k ; t\right)+b x M_{n}^{\prime}\left(x_{o}, k ; t\right)=-n M_{n-1}\left(x_{o}, k ; t\right)
$$

or equivalently; 


$$
M_{n}^{\prime \prime}\left(x_{o}, k ; t\right)+\frac{b}{a} M_{n}^{\prime}\left(x_{o}, k ; t\right)=-\frac{n}{a x} M_{n-1}\left(x_{o}, k ; t\right)
$$

where $M_{n}^{\prime}\left(x_{0}, k ; t\right)$ and $M_{n}^{\prime \prime}\left(x_{o}, k ; t\right)$ are the first and second derivatives of $M_{n}\left(x_{o}, k ; t\right)$ with respect to $x\left(x_{o} \leq x \leq k\right)$, with appropriate boundary conditions for $n=1,2,3 \ldots$. Note that $M_{0}\left(x_{0}, k ; t\right)=1$.

Now, rewrite Eq. (5), as

$$
M_{n}^{\prime \prime}\left(x_{o}, k ; t\right)=-\frac{n}{a x} M_{n-1}\left(x_{o}, k ; t\right)-\frac{b}{a} M_{n}^{\prime}\left(x_{o}, k ; t\right) .
$$

Let $\Delta$ be the difference operator. Then we defined the first order difference of $M_{n}\left(x_{o}, k, t\right)$ as follows:

$$
\Delta M_{n}\left(x_{o}, k ; t\right)=M_{n+1}\left(x_{o}, k ; t\right)-M_{n}\left(x_{o}, k ; t\right)
$$

(cf. Kelley and Peterson (1991)).

Note that Eq. (6) can be approximated by

$$
M_{n}^{\prime \prime}\left(x_{o}, k ; t\right)=-\frac{n}{a x} M_{n-1}\left(x_{o}, k ; t\right)+\frac{b}{a} \Delta M_{n}\left(x_{o}, k ; t\right)
$$

By substituting from Eq. (7) in Eq. (8) we get:

$$
M_{n}^{\prime \prime}\left(x_{o}, k ; t\right)=-\frac{n}{a x} M_{n-1}\left(x_{o}, k ; t\right)+\frac{b}{a} M_{n}\left(x_{o}, k ; t\right)-\frac{b}{a} M_{n+1}\left(x_{o}, k ; t\right)
$$

Now, we will use the matrix theory to solve the differential equation defined in Eq. (9). If we let

$$
\vec{M}\left(x_{o}, k ; t\right)=\left[M_{1}\left(x_{o}, k ; t\right), M_{2}\left(x_{o}, k ; t\right),,,,\right]
$$

Then we get

$$
\frac{d^{2} \vec{M}\left(x_{o}, k ; t\right)}{d x^{2}}=A \vec{M}\left(x_{o}, k ; t\right)
$$

where

$$
\mathrm{A}=\left[\begin{array}{ccccc}
\frac{b}{a} & -\frac{b}{a} & 0 & 0 & \ldots \\
-\frac{2}{a x} & \frac{b}{a} & -\frac{b}{a} & 0 & \ldots \\
0 & -\frac{3}{a x} & \frac{b}{a} & -\frac{b}{a} & \ldots \\
0 & 0 & -\frac{4}{a x} & \frac{b}{a} & \ldots \\
\vdots & \vdots & \vdots & \vdots & \ddots
\end{array}\right] .
$$

Now let

$$
\frac{d \vec{M}\left(x_{o}, k ; t\right)}{d x}=\vec{R}\left(x_{o}, k ; t\right)
$$

This implies

$$
\frac{d^{2} \vec{M}\left(x_{o}, k ; t\right)}{d x^{2}}=\frac{d \vec{R}\left(x_{o}, k ; t\right)}{d x} .
$$


Apply to Eq. (10), we get

$$
\frac{d}{d x}\left[\begin{array}{l}
\vec{R}\left(x_{o}, k ; t\right) \\
\vec{M}\left(x_{o}, k ; t\right)
\end{array}\right]=\left[\begin{array}{cc}
O & A \\
I & O
\end{array}\right] \cdot\left[\begin{array}{l}
\vec{R}\left(x_{o}, k ; t\right) \\
\vec{M}\left(x_{o}, k ; t\right)
\end{array}\right]
$$

where $I$ is the identity matrix and $O$ is the zero matrix.

Thus the solution of the system of equations in Eq. (13) is given by

$$
\left[\begin{array}{l}
\vec{R}\left(x_{o}, k ; t\right) \\
\vec{M}\left(x_{o}, k ; t\right)
\end{array}\right]=e^{\left[\begin{array}{cc}
O & A^{*} \\
D & o
\end{array}\right]} \cdot\left[\begin{array}{l}
\vec{R}\left(x_{o}, k ; t\right) \\
\vec{M}\left(x_{o}, k ; t\right)
\end{array}\right]
$$

where $D=\left[d_{i j}\right] ; i ; j \geq 1$ is the diagonal matrix with entries

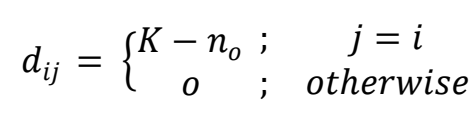

and $A^{*}=\left\{\alpha_{i j}\right\} ; i, j \geq 1$ is the matrix with entries

$$
a_{i j}=\left\{\begin{array}{c}
-\frac{i}{\alpha} \ln \left(\frac{k}{x_{o}}\right) ; j=i-1 \\
\frac{b}{a}\left(k-x_{o}\right) ; j=i \\
-\frac{b}{a}\left(k-x_{o}\right) ; j=i+1 \\
o ; \text { otherwise. }
\end{array}\right.
$$

Note that the matrix $e^{B}$ where $B=\left[\begin{array}{cc}O & A^{*} \\ D & o\end{array}\right]$ is defined by

$$
e^{B}=I+B+\frac{B^{2}}{2 !}+\frac{B^{3}}{3 !}+\cdots
$$

This series is convergent since it is a Cauchy operator of Eq. (2.6) (cf. Zeifman (1991)).

\section{Conclusion}

In conclusion the advantage of this technique is to use the difference equation to approximate the ordinary differential equation since it is the discretization of the ODE. Also, the system of the solutions in equations (14) gives an explicit solution to the first-passage time moments for the birth and death GNP diffusion process to a fixed determined value of GNP (constant single absorbing barrier). This increases the applicability of the diffusion process in stochastic modeling in the area of economics.

\section{References}

A. J. Alawneh and B. M. Aleideh (2002). Moment approximation of the first-passage time for the Ornstein-Uhlenbeck process. Intern. Math. J. Vol. 1 (3), pp. 255-258.

I. F. Blake and W. C. Lindsey (1973). Level crossing problems for random processes, IEEE Trans. Information Theory, Vol. 19, pp. 295-315.

D. R. Cox and H. D. Miller (1965). The Theory of Stochastic processes; Methuen, London.

D. Darling and A. J. F. Siegert (1953). The first-passage problem for a continuous Markov process, Ann. Math. Statist. Vol. 24 (1953), pp. 624-639.

J. Durbin (1971). Boundary-crossing probabilities for the Brownian motion and Poisson processes and techniques for computing the power of Kolmogorov - Smirnov test. J. Appl. Prob. Vol. 8, pp. 431-453. 
W. J. Ewens (1979). Mathmatical Population Genetics. Springer-Verlag, Berlin.

B. Ferebee (1982). The tangent approximation to one-sided Brownian exit densities, Z. Wahrscheinlichkeitsth, Vol.61, pp. 309-326.

R. Gutierrez, L. Ricciardi, P. Roman and F. Terres (1997). First-passage time densities for time-nonhomogeneous diffusion processes. J. Appl. Prob. , Vol. 34, pp. 623-631.

R. Gutierrez, P. Roman and F. Terres (1999). Inference and first-passage times for the lognormal diffusion process with exogenous factors: Application to modeling in economics. Appl. Stochastic Models Bus. Ind. Vol. 15, pp. 325-332.

D. L. Iglehart (1965). Limiting diffusion approximation for the many server queue and the repairman problem. J. Appl. Prob. , Vol.2 , pp. 429-441.

S. Karlin and H. M. Taylor (1981). A second course in stochastic processes, Academic Press, New York.

W. G. Kelly and A. C. Peterson (1991). Difference Equations: An Introduction with Applications, Academic Press, New York.

D. R. McNeil (1970). Integral Functionals of birth and death processes and related limiting distributions. Ann. Math. Statist. , Vol. 41, pp. 480-485.

D. R. McNeil and S. Schach (1973). Central limit analogues for Markov population processes. J. R. Statist. Soc. B. , Vol. 35, pp. $1-23$.

M. U. Thomas (1975). Some mean first passage time approximations for the Ornstein-Uhlenbeck process, F. Appl. Prob. , Vol. 12, pp. 600-604.

H. C. Tuckwell and F. Y. M. Wan (1984). First passage time of Markov processes to moving barriers. J. Appl. Prob. , Vol. 21, pp. 695-709.

A. I. Zeifman (1991). Some estimates of the role of convergence for birth and death processes. J. Appl. Prob. , Vol. 28, pp. 268-277. 